\title{
On the Uplink Capacity of Cellular CDMA and TDMA over Nondispersive Channels
}

\author{
(1) Alcatel Access Systems Division \\ 5 , rue Noel-Pons \\ 92734 Nanterre Cedex, France \\ Hikmet.Sari@alcatel.fr
}

Hikmet Sari $^{(1)}$, Heidi Steendam ${ }^{(2)}$, Marc Moeneclaey ${ }^{(2)}$

\begin{abstract}
We investigate the interuser interference on the uplink channel (from users to base station) in cellular code-division multiple access (CDMA) and time-division multiple access (TDMA) systems. The results indicate that for the same total bandwidth occupancy and maximum number of users per cell, interuser interference is approximately $10 \mathrm{~dB}$ higher in CDMA in fully-loaded cells. The implication of these results is that for the same total bandwidth and number of users per cell, TDMA gives superior bit error rate (BER) performance, as confirmed by our numerical results on additive white Gaussian noise (AWGN) channels as well as on flat Rayleigh fading channels. Alternatively, CDMA needs approximately 10 times more bandwidth than TDMA to achieve the same signalto-interference $(\mathrm{S} / \mathrm{I})$ ratio and bit error rate performance in fully loaded cells.
\end{abstract}

\section{INTRODUCTION}

Code-division multiple access (CDMA) has become very popular over the past few years, not only in mobile and personal communications, but also in other applications including fixed wireless access and satellite systems. While it is undeniable that this multiple access technique has the virtue of easing frequency planning, the capacity of CDMA and its comparison to timedivision multiple access (TDMA) has been a very controversial issue often dominated by commercial interests. In addition, comparisons are usually made between systems in which TDMA or CDMA is only one ingredient among many others. The consequence of this is that the capacity of CDMA is still not a well understood issue which needs further clarification.

The purpose of the present paper is to compare the uplink capacity of CDMA and TDMA. Before describing the CDMA scheme considered in this study, note that there are essentially two basic CDMA techniques: The first one directly derives from directsequence spread-spectrum (DS-SS) systems which were
${ }^{(2)}$ Communications Engineering Laboratory University of Ghent, St. Pietersnieuwstraat 41 B9000 Gent, Belgium Marc.Moeneclaey@telin.rug.ac.be

originally deviced for military communication systems [1], [2]. DS-SS has two attractive features for those applications: The first feature is the low intercept probability which results from the fact that a DS-SS signal is virtually buried in background noise both in the time and in the frequency domains. The second feature is the robustness of DS-SS signals to intentional or unintentional jamming. It is obvious that the spreading sequence in these applications must be pseudo-random and difficult to replicate by an unauthorized user, because if such a user knows and can use the spreading sequence, the whole process becomes useless. In the sequel, we will refer to CDMA systems which use pseudo-random (pseudo-noise) spreading sequences as pseudo-noise CDMA (PN-CDMA).

The second basic CDMA technique is also based on direct sequence spectral spreading, but uses a set of orthogonal sequences, e.g., Walsh-Hadamard (WH) sequences [3], instead of pseudo-noise sequences. There is no randomness in this case, and the spreading sequence repeats itself from one symbol to the next. Furthermore, since the spreading sequences are orthogonal, there is no mutual interference between different user signals in this technique.

The paper is organized as follows: In the next section, we briefly describe TDMA and CDMA, and recall their basic features. Next, in Section 3, we present the cellular TDMA and CDMA schemes that we consider in our analysis, and we compute their interuser interference on the uplink. Section 4 reports some numerical results, and Section 5 gives our conclusions.

\section{A BRIEF REVIEW OF TDMA AND CDMA}

TDMA consists of sharing a data stream between different users by assigning to them different time slots. For simplicity, we will focus here on a simple TDMA scheme in which the data stream is formatted into frames 
of $\mathrm{N}$ time slots and each user gets one time slot per frame. That is, the channel resources are equally shared between $\mathrm{N}$ different users. The total bandwidth of the transmitted signal in this scheme is $\mathrm{N}$ times the bandwidth which would be needed by a single user if this user were alone on the channel. Let $\mathrm{R}$ designate the symbol rate of each user, and $\mathrm{W} \mathrm{Hz}$ the bandwidth required to transmit this symbol rate in single-user transmission. The TDMA scheme at hand thus transmits a symbol rate of NR baud using a bandwidth of NW Hz. Of course, the overhead needed for signal framing is neglected in this simple example.

An equivalent OCDMA scheme assigns a periodic sequence of length $\mathrm{N}$ to each user, where all sequences are mutually orthogonal. The period of the sequences coincides with the symbol interval, giving thus $\mathrm{N}$ chips per symbol. This process spreads the transmitted signal bandwidth by a factor of $N$. Since the number of orthogonal sequences of length $\mathrm{N}$ is exactly $\mathrm{N}$, this OCDMA scheme can accommodate $\mathrm{N}$ users. In other words, exactly as in TDMA, OCDMA can accommodate $\mathrm{N}$ users when the available bandwidth is $\mathrm{N}$ times that required by one user, and this is achieved without any mutual interference.

We now analyze PN-CDMA. Let $\mathrm{p}_{\mathrm{k} l}$ designate the portion of the $k$ th user's PN sequence that is used during the $l$ th symbol period. Since the $\mathrm{p}_{\mathrm{k} l}$ sequences are uncorrelated PN sequences of length $\mathrm{N}$, we have

$$
E\left(p_{k l} p_{k l}\right)=N \delta_{l l}
$$

where $\delta$ denotes the Kronecker delta which takes the value of 1 for $l=l$ ' and of 0 for $l \neq l$ '. Therefore, the correlator preceding the decision circuit at the receiver gives a useful signal value of $\mathrm{N}$ (times the transmitted symbol), while the average interference value from any other user is 0 . The mean-squared value of this interference is

$$
E\left\{\left(p_{k l} p_{k l}\right)^{2}\right\}=N
$$

The signal-to-interference (S/I) power ratio is therefore $\mathrm{N}^{2} / \mathrm{N}=\mathrm{N}$, or equivalently the interference power normalized by the useful signal power is $1 / \mathrm{N}$. Interferences from different users add up, and the normalized interference power becomes $\mathrm{n} / \mathrm{N}$ when the number of users is $n$. If we assume that the number of active users is $\mathrm{N}$, then each user will get interference from the other $\mathrm{N}-1$ users, and the total normalized interference power will be $(\mathrm{N}-1) / \mathrm{N}$, which indicates that for large $\mathrm{N}$, the interference power is as large as the useful signal power. Obviously, no operation is possible at such a high interference level even with the best error correction codes, and this means that PN-CDMA accommodates a much smaller number of users than TDMA and OCDMA. If the interference power is to be limited to for example $20 \%$ of the signal power, then only N/5 users can be accommodated, and this represents a capacity decrease by a factor of 5 with respect to TDMA and OCDMA. The capacity of PNCDMA is therefore not a fixed number; It depends on the interference level that one is prepared to tolerate. To summarize the single-cell case, while both TDMA and OCDMA can accommodate $\mathrm{N}$ users without any mutual interference on a channel whose bandwidth is $\mathrm{N}$ times that required by a single user, PN-CDMA leads to interuser interference as soon as there are 2 users on the channel, and the interference power is proportional to the number of users. As a result, the number of users that can be accommodated is substantially lower than $\mathrm{N}$ if the performance is to be kept at an acceptable level.

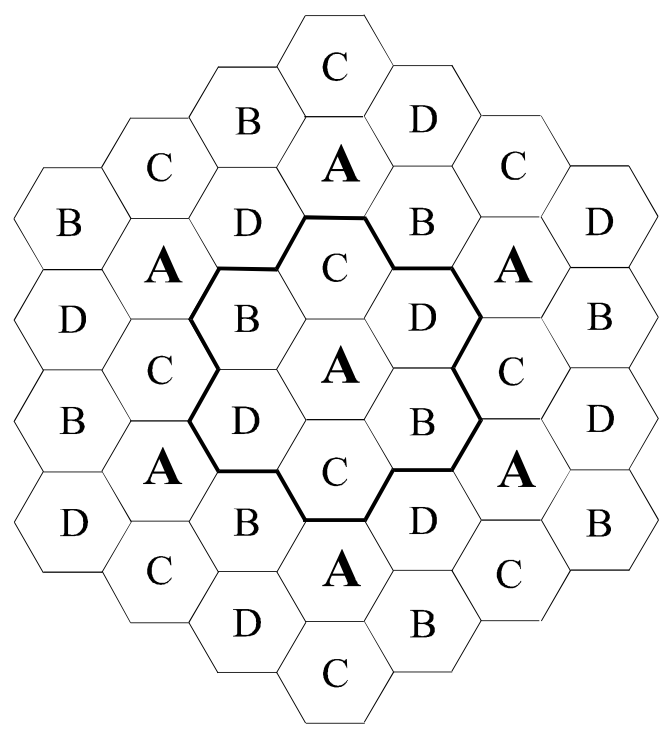

Fig. 1 : Cell structure for frequency reuse factor of 4 .

\section{FREQUENCY REUSE AND INTERUSER INTERFERENCE}

In the previous section, we analyzed the capacity of CDMA in a single cell. In real-world applications, communication systems typically operate in a multicell environment, and the basic question is the achievable frequency reuse factor among cells. We will consider here a hexagonal cell pattern commonly used in mobile radio systems. In TDMA, two adjacent cells can not use the same carrier frequency, and therefore the smallest number of frequencies is 3 . If the $\mathrm{S} / \mathrm{I}$ ratio with this 
frequency reuse factor is not sufficient, a frequency reuse factor of 4 or 7 can be used instead. Such frequency reuse patterns are commonly found in stateof-the-art textbooks on digital mobile radio systems [4]. In what follows, we will assume a frequency reuse factor of 4, following the cell pattern of Fig. 1. Each cell in this figure is labeled with a letter which indicates the frequency (or group of frequencies) used. If we consider an A-cell, the 6 cells surrounding it are of type B, C, D, $\mathrm{B}, \mathrm{C}$, and $\mathrm{D}$, respectively. If $\mathrm{R}$ designates the cell radius (i.e. the radius of the largest circle that fits inside the hexagonal cell), then the distance between two base stations using the same frequency is $4 \mathrm{R}$. Furthermore, each cell has 6 nearest neighbors which employ the same frequency.

\section{A. TDMA}

Let us now examine the upstream (from users to base station) interference problem in TDMA in this environment. Interference is obviously limited to cells that employ the same carrier frequency, i.e., an A-cell gets interference only from other A-cells, a B-cell from other B-cells, and so forth. If the clocks of different cells are mutually synchronized, then the interference will be limited to users that share common time slots. In other words, there is a one-to-one correspondence between interfering users in that case. However, it is quite unlikely that clocks of different cells will be synchronized in practice. In the presence of clock frequency offset, a given user will sequentially interfere with all other users of the interfering cells, the interference resembling a periodic impulse noise. To proceed further, we assume that all base stations transmit the same signal level, and that the transmit power control in the upstream direction is perfect so that a base station receives the same signal level from all users in its cell.

Now we compute the S/I ratio assuming that the propagation loss is proportional to the fourth power of the distance. Let us consider two cells that operate at the same frequency, and assume that the cells are fully loaded ( $\mathrm{N}$ active users per cell). The useful signal, received during a given symbol interval by the base station of the first cell, is disturbed by the user in the second cell that transmits during the same symbol interval. The interference caused by that particular user depends on its distance $d$ to the base station of the first cell, and on its distance $r$ to its own base station. Assuming ideal power control, the transmit power of a user at a distance $r$ from its base station is proportional to $r^{4}$, so that this base station receives a power that is independent of the user position. Normalizing by one the energy that a base station receives from its user during the considered symbol interval, the energy received during the same symbol interval from the disturbing user at distance $\mathrm{d}$ is equal to $(\mathrm{r} / \mathrm{d})^{4}$, with $\mathrm{r}$ denoting the distance between the interfering user and its own base station. The average interfering energy per symbol is obtained by averaging $(\mathrm{r} / \mathrm{d})^{4}$ over the position of the interfering user within its cell. Denoting by D the distance between the base stations of the two considered cells, one obtains

$$
d^{2}=D^{2}+2 D r \cos \theta+r^{2}
$$

where $\theta$ is the angle indicated in Fig. 2. When the cell radius is equal to $R$, the average interfering energy per symbol caused by one interfering cell, whose base station is at distance D from the considered base station, is given by

$$
I_{\text {cell }}(D)=\frac{2}{3 \sqrt{3} R^{2}} \iint_{\text {cell }} \frac{r^{4}}{\left(D^{2}+2 D r \cos \theta+r^{2}\right)^{2}} r d r d \theta
$$

Taking into account only the nearest 6 interfering cells (with $\mathrm{D}=4 \mathrm{R}$ ), the total interference $\mathrm{I}$ is given by $\mathrm{I}=$ 6.I $\mathrm{I}_{\text {cell }}(4 \mathrm{R})$. Evaluating (4) by means of a numerical integration routine, the uplink $\mathrm{S} / \mathrm{I}$ ratio is $\mathrm{S} / \mathrm{I}=82.8$, or $\mathrm{S} / \mathrm{I}=19.2 \mathrm{~dB}$. This uplink $\mathrm{S} / \mathrm{I}$ ratio holds only when the considered time slot is also used in all of the 6 nearest cells that employ the same frequency. Otherwise, the S/I ratio will be higher.

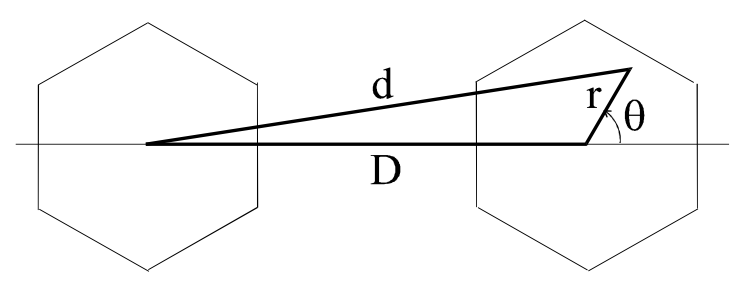

Fig. 2 : Illustration of interfering user location

\section{B. Hybrid CDMA}

In order to avoid the large intracell interference that occurs in a pure PN-CDMA system, we consider hybrid CDMA with an intracell OCDMA and an intercell PNCDMA. That is, the transmitted signal is first spread through multiplication by a WH sequence with $\mathrm{N}$ chips per symbol. Then, a PN sequence is overlayed either without further spreading (i.e., with the same chip rate) or with further spreading by a factor of 2 or 4 . All users of a cell employ the same $\mathrm{PN}$ sequence, and $\mathrm{N}$ orthogonal sequences are reused in each cell. This kind 
of multiple access is used in IS-95 [4] and in current proposals for the future universal mobile telecommunications systems (UMTS) [5]. In this scheme, there is no interference between different users in the same cell, but a given user interferes with all users of all other cells. If the chip rates of the orthogonal and PN sequences are the same, the mutual interference between two users is $1 / \mathrm{N}$ (attenuated by a factor which is function of the user location). If the number of PN chips per WH chip is 4 , then the mutual interference is $1 / 4 \mathrm{~N}$, and in terms of bandwidth occupancy the resulting system is equivalent to the TDMA system with a frequency reuse factor of 4 considered above. The basic question is whether this system can accommodate as many users as does TDMA and at what performance level.

In what follows, we will evaluate the total uplink interference level in hybrid CDMA assuming that the PN sequence has 4 chips per WH chip (so that the bandwidth is the same as for TDMA with a reuse factor of 4). We take into account only the interference coming from the 6 nearest cells (with $\mathrm{D}=2 \mathrm{R}$ ), the 6 secondnearest cells (with $D=\sqrt{12} R$ ), and the 6 third-nearest cells (with $D=4 R$ ). When the number of users per cell and the number of chips per symbol are equal to $\mathrm{n}$ and $4 \mathrm{~N}$, respectively, the uplink S/I ratio is given by

$$
\frac{S}{I}=\frac{4 N}{6 n\left(I_{\text {cell }}(2 R)+I_{\text {cell }}(\sqrt{12} R)+I_{\text {cell }}(4 R)\right)}
$$

which yields $\mathrm{S} / \mathrm{I}=2.40(4 \mathrm{~N} / \mathrm{n})$. For $\mathrm{n}=\mathrm{N}$, we obtain $\mathrm{S} / \mathrm{I}$ $=9.8 \mathrm{~dB}$. This is $9.4 \mathrm{~dB}$ worse than the uplink $\mathrm{S} / \mathrm{I}$ ratio in the equivalent TDMA scheme.

\section{PERFORMANCE ANALYSIS}

In this section, we will report some numerical results comparing bit error rate (BER) performance of TDMA and hybrid CDMA with and without coding, and both at low and high user densities in different cells. The TDMA system has a frequency reuse factor of 4 and $\mathrm{N}$ users per cell each having the same bit rate as the users in the CDMA scheme to which it is compared. The CDMA system has 4N chips per symbol, and $\mathrm{N}$ orthogonal sequences are allocated to each cell, i.e., the maximum number of users per cell is N. The TDMA and CDMA systems considered obviously require the same total bandwidth. For these two systems, numerical results are reported in Figs. 3 - 5 assuming binary phaseshift keying (BPSK) modulation and $\mathrm{N}=64$.

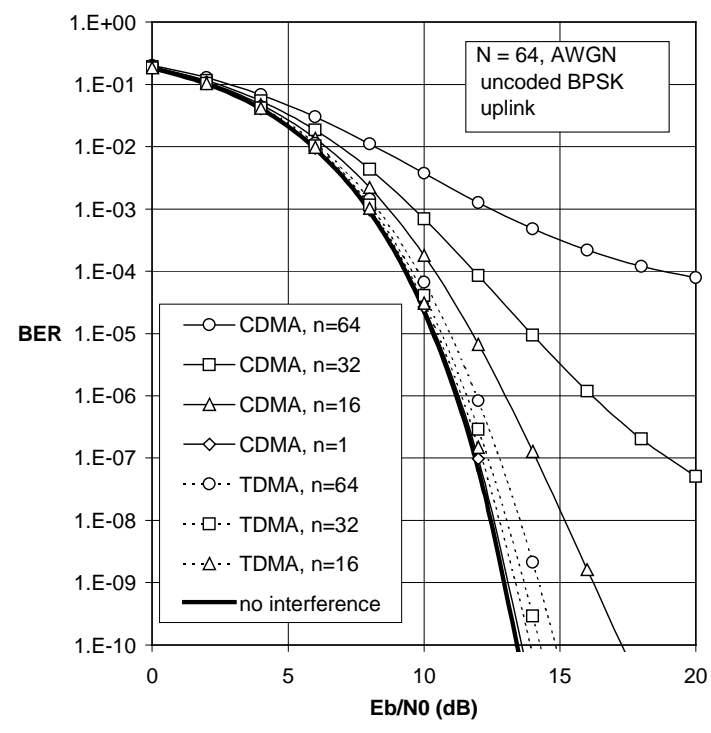

Fig.3 : Uplink BER performance for uncoded transmission over AWGN channel

Fig. 3 gives the BER performance on additive white Gaussian noise (AWGN) channels in the absence of channel coding. We observe that with $n=32$ or 64 users, CDMA exhibits a BER floor, and that the signalto-noise ratio (SNR) degradation is strong even with 16 users. As for TDMA, its SNR degradation is modest and remains below $1 \mathrm{~dB}$ at the BER of $10^{-8}$ even with $\mathrm{n}=$ 64. Next, Fig. 4 shows the results obtained using the industry-standard convolutional code with rate $1 / 2$ and constraint length $K=7$. The degradations are smaller in this case, but here too TDMA outperforms CDMA. Finally, Fig. 5 shows the results over a flat Rayleigh fading channel with the same convolutional code as in Fig. 4. Ideal interleaving is assumed so that channel attenuation is uncorrelated from one symbol to the next. Note that the SNR degradation of CDMA is significantly higher with respect to the AWGN channel case, while the degradation of TDMA remains negligible.

Before closing this section, let us point out that it is not surprising to see TDMA outperform CDMA, particularly when the propagation loss is proportional to the fourth-power of the distance. The reason is that interference in TDMA with a frequency reuse factor of 4 originates from cells whose centers are at a distance of 4R or higher from the serving base station, whereas users in CDMA are faced with interference from all neighboring cells whose centers are only at a distance $2 \mathrm{R}$, and second-nearest neighbor cells whose centers are at a distance $\sqrt{12} \mathrm{R}$. Interference from these cells is 
much less attenuated than that from the third-nearest neighbor cells which interfere in TDMA.

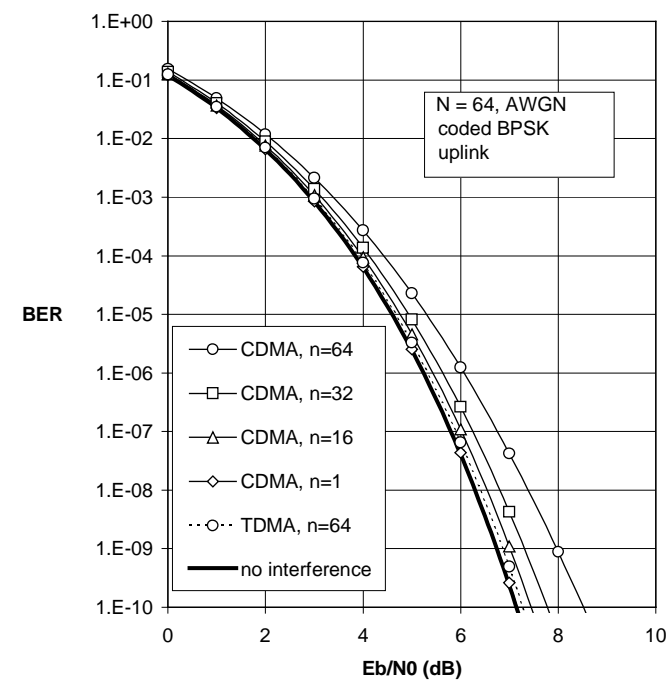

Fig. 4 : Uplink BER performance for coded transmission over AWGN channel

\section{CONCLUSIONS}

We have compared two popular cellular system concepts with at most $\mathrm{N}$ users per cell. One of them is based on TDMA with a frequency reuse factor of 4 between cells. The other is based on hybrid CDMA with $4 \mathrm{~N}$ chips per symbol and $\mathrm{N}$ orthogonal sequences per cell. Using a propagation model in which the signal attenuation is proportional to the fourth-power of the distance, we have shown that on the upstream channel, TDMA has a S/I ratio advantage of approximately 10 $\mathrm{dB}$ over CDMA. Our BER performance results on AWGN channels and flat Rayleigh fading channels confirmed the superiority of TDMA, particularly at high user densities. At small user densities, the interference power is small compared to the noise power, and therefore TDMA and CDMA have similar BER performance.

Note that CDMA and TDMA have a similar BER performance when they operate at the same S/I ratio. Since there is a gap of $10 \mathrm{~dB}$ between the two systems considered in this paper, hybrid CDMA needs to use $40 \mathrm{~N}$ chips per symbol to achieve the same S/I ratio as the TDMA with a frequency reuse factor of 4 . In turn, such a CDMA system requires 10 times the bandwidth of TDMA which accommodates the same number of users per cell and achieves the same S/I ratio. This result may seem surprising, because it contradicts the usual claims that CDMA achieves a higher capacity than
TDMA. But the fact that CDMA uses the same frequency in all cells does not imply that it can support a higher number of users in a given geographic area and a given total frequency bandwidth, and the results reported in the present paper show that the opposite is true when the same S/I ratio is set for both schemes.

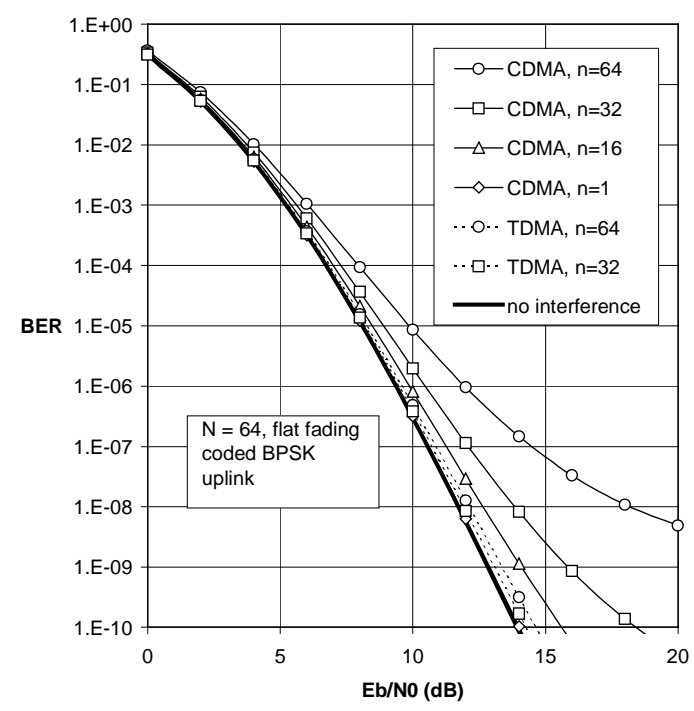

Fig. 5 : Uplink BER performance for coded transmission over flat Rayleigh fading channel

\section{REFERENCES}

[1]R. C. Dixon, "Spread Spectrum Systems," 2nd Edition, John Wiley and Sons, New York, 1984.

[2] J. G. Proakis, "Digital Communications," 2nd Edition, McGraw Hill, New York, 1989.

[3] N. Ahmed and K. R. Rao, "Orthogonal Transforms for Digital Signal Processing," Springer-Verlag, BerlinHeidelberg, 1975.

[4] T. S. Rappaport, "Wireless Communications: Principles and Practice," IEEE Press, NewYork, and Prentice Hall, New Jersey, 1996.

[5] F. Adachi, M. Sawahashi, and H. Suda, "Wideband DS-CDMA for Next-Generation Mobile Communications Systems," IEEE Communications Magazine, vol. 36, no. 9, pp. 56-69, September 1998. 